\title{
Microsaccades under monocular viewing conditions
}

\author{
Wilhelm Bernhard Kloke, Wolfgang Jaschinski and Stephanie Jainta \\ Leibniz-Institut für Arbeitsforschung an der TU Dortmund
}

\begin{abstract}
Among the eye movements during fixation, the function of small saccades occuring quite commonly at fixation is still unclear. It has been reported that a substantial number of these microsaccades seem to occur in only one of the eyes. The aim of the present study is to investigate microsaccades in monocular stimulation conditions. Although this is an artificial test condition which does not occur in natural vision, this monocular presentation paradigm allows for a critical test of a presumptive monocular mechanism of saccade generation. Results in these conditions can be compared with the normal binocular stimulation mode. We checked the statistical properties of microsaccades under monocular stimulation conditions and found no indication for specific interactions for monocularly detected small saccades, which might be present if they were based on a monocular physiological activation mechanism.
\end{abstract}

Keywords: Microsaccades; Binocular Coordination; Eye Movements; Fixation

\section{Introduction}

When the eyes are fixating a visual target, they are continuously moving in several ways which can be differentiated into three categories: (1) Tremor is a fast irregular movement with a high frequency spectrum above $50 \mathrm{~Hz}$ and an amplitude of about the size of one cone in the fovea, (2) drifts have a low frequency and an amplitude of a dozen cones, and (3) microsaccades are, by definition, those saccades which are not intended to bring a new target into the center of the fovea, but rather occur when maintaining the current fixation. Microsaccades happen quite frequently, about two per second on average. With respect to their kinematic traits, they resemble other saccades of the same size (Zuber, Stark, \& Cook, 1965), e.g. they have the same relation of width and velocity ("main sequence"). Just as ordinary saccades, microsaccades induce a temporary elevation of perception thresholds, the saccadic suppression (Zuber \& Stark, 1966).

The specific physiological function of microsaccades is not agreed upon, despite a vehement dispute since more than 40 years. As an extreme position, Kowler and Steinman (1980) denied that microsaccades are of any use. On the other hand, recently the interest in the study of microsaccades has been revived, mostly because the microsaccadic activity seems to reveal some insight into the inner working mechanisms of the mind, see (Martinez-Conde \& Macknik, 2007) and (Engbert \& Kliegl, 2003a). As shown by the broad review of Martinez-Conde, Macknik, and Hubel (2004), possible physiological effects include the prevention of fading in the visual periphery, fine control of fixation position or enhancement of acuity. For some of these effects, experimental support has been found, e.g. MartinezConde et al. (2006) showed that the fading of Troxler figures is correlated to lowered microsaccade activity before the loss of perception. Troncoso, Macknik, and Martinez-Conde (2008) extended this fading prevention for second-order visibility, using artificial scotomas. Bridgeman and Palca (1980) pointed out that saccadic suppression caused by microsaccades seems to be detrimental to the perception process. Therefore stronger reasons for the existence of microsaccades are still investigated. Kadyrov and Petrou (2004) showed that computational reconstruction of nonhomogeneously sampled images is vastly improved by integrating over a set of slightly shifted images. Nonhomogenuous sampling is the case in the retina, especially in the near periphery of the fovea. It is not clear, however, how such integration could be accomplished in the real physiological system.

Filin (1997) proposed a theory of automatic saccadic activity, which may partly be an explanation for the existence of microsaccades. He views the continuous initiation of saccades as an autonomous process similar to breathing, which is active at any time, but may sometimes be modulated by intention. In this context, microsaccades are just the relics of this process at times when the intent to fixate would be defeated by saccades of larger size. The idea of autonomous saccade generation has been proposed by other authors also, e.g. Engbert and Kliegl (2001), Richter, Engbert, and Kliegl (2008). Hafed, Goffart, and Krauzlis (2009) showed that microsaccades are likely generated in the superior colliculus, which was already known to be important for the generation of voluntary saccades. Rolfs, Kliegl, and Engbert (2008) provided fur- 
ther evidence for the essential similarity of the generation of microsaccades and saccades. The view of a continous microsaccade-saccade continuum was extended to free-viewing contexts by Otero-Millan, Troncoso, Macknik, Serrano-Pedraza, and Martinez-Conde (2008).

Only limited research investigated the question whether microsaccades occur synchronously in both eyes or not. Under normal viewing conditions, the intentional movements of both eyes are tightly coupled by Hering's law. Therefore, the 2-eye system is better viewed as a single organ system, called cyclopic eye, with a vergence subsystem which manages the submovements caused by the variation of the vergence angles between the two visual axes at varying viewing distances. Saccades are actions of the cyclopic eye, though they are regularly accompanied with temporary vergence change. Earlier researchers, such as Krauskopf, Cornsweet, and Riggs (1960), emphasized that microsaccades occur synchronously in both eyes. This was confirmed by Møller, Laursen, Tygesen, and Sjølie (2002) for a data set from 10 subjects measured with a video-based Eye-Link system. In contrast, Engbert and Kliegl (2003b) reported that using an objective algorithm for microsaccade detection, they found a remarkably high number of microsaccades which seemed to occur in only one of the eyes, namely about $40 \%$. Further, they reported some statistical differences between monocular and binocular saccades; binocular saccades were predominantly horizontally oriented, monocular saccades both vertically and horizontally and of smaller size. So far, no convincing explanation for this finding seems to have been found, though results by Zhou and King (1998) imply a basically monocular mechanism for eye movement generation. The implication of their finding regarding Hering's law was questioned by Mays (1998). In most studies, e. g. (Otero-Millan et al., 2008), using an automatic algorithm, microsaccades that appeared monocular have therefore been excluded before further evaluation, in order to reduce the amount of potential noise.

Anyway, if microsaccades have a function which is related to the content of visual perception and there is a specific monocular saccade generation mechanism, then some statistical properties of observed microsaccades may depend on (1) whether stimuli are presented to both eyes or to one eye only, and (2) for the case of monocularly detected saccades, whether the eye on which the saccade occurred, is the stimulated eye or the other one. Without a sound theory of microsaccades, it is not possible to predict the direction and size of such an effect. Otero-Millan et al. (2008) reported that in free-viewing tasks at a blank screen, microsaccade production was lower than in tasks with attentional targets. However, autonomous saccades tend to have a larger size under conditions of no or weak stimulation, as Filin and Ananin (1973) reported for the cases of blindness, pathologic vision and sleep. It may well be the case that there are different effects present and oppositely directed effects may cancel each other.

Therefore it is the aim of the present study to investigate microsaccades under conditions where different visual content is presented to the two eyes. We compare microsaccade data from an experiment that included (1) phases in which stimuli were presented to the right or left eye only and (2) phases with stimuli with binocular presentation, which is the conventional viewing condition.

\section{Method}

\section{Experimental setup}

We used the data from an experiment designed to study binocular coordination of saccades in a simplified reading process (Jaschinski, Kloke, \& Jainta, 2009). The experiment was carried out at a mirror stereoscope, on which stimulus data are generated on two CRT monitors separately for each eye and viewed on mirrors.

Apparatus. Eye movements were measured binocularly using Eyelink II cameras mounted at a head- and chinrest. The manufacturer's specification of the absolute measurement precision (based on the original Eyelink II calibration procedure), is not sufficient for measuring the difference in eye positions exactly enough as required for objective fixation disparity analyses. Therefore, our own monocular calibration routine was included. The quality of the calibration can be judged from the inner consistence of these calibration data and their replication. In the context of the present paper the quality of the calibration process as such plays a lesser role because only relative data in a short time range are used. For the conclusions made here it is only important, that the instrument gain is only slowly varying in time and is roughly correctly estimated, so that variance introduced from calibration errors does not dominate the results. In this study, the calibration measurements are used as data for the monocular condition.

Binocular phases. The subjects had to fixate on 3 targets from left to right in turn, which were positioned in 5 degree steps horizontally at $60 \mathrm{~cm}$ viewing distance. Three quasi-words consisting of three letters each were statically presented to both eyes. The subjects were asked to fixate the middle letter of each quasi-word in reading-like order. At this position, dichoptic monocular nonius lines were flashed above and below the middle letter in order to measure the fixation disparity by subjective judgement. The subject responded by choosing the left or right mouse switch. In a variant condition of this experiment, only the middle of the target positions was used, thus no saccades between targets were required. 


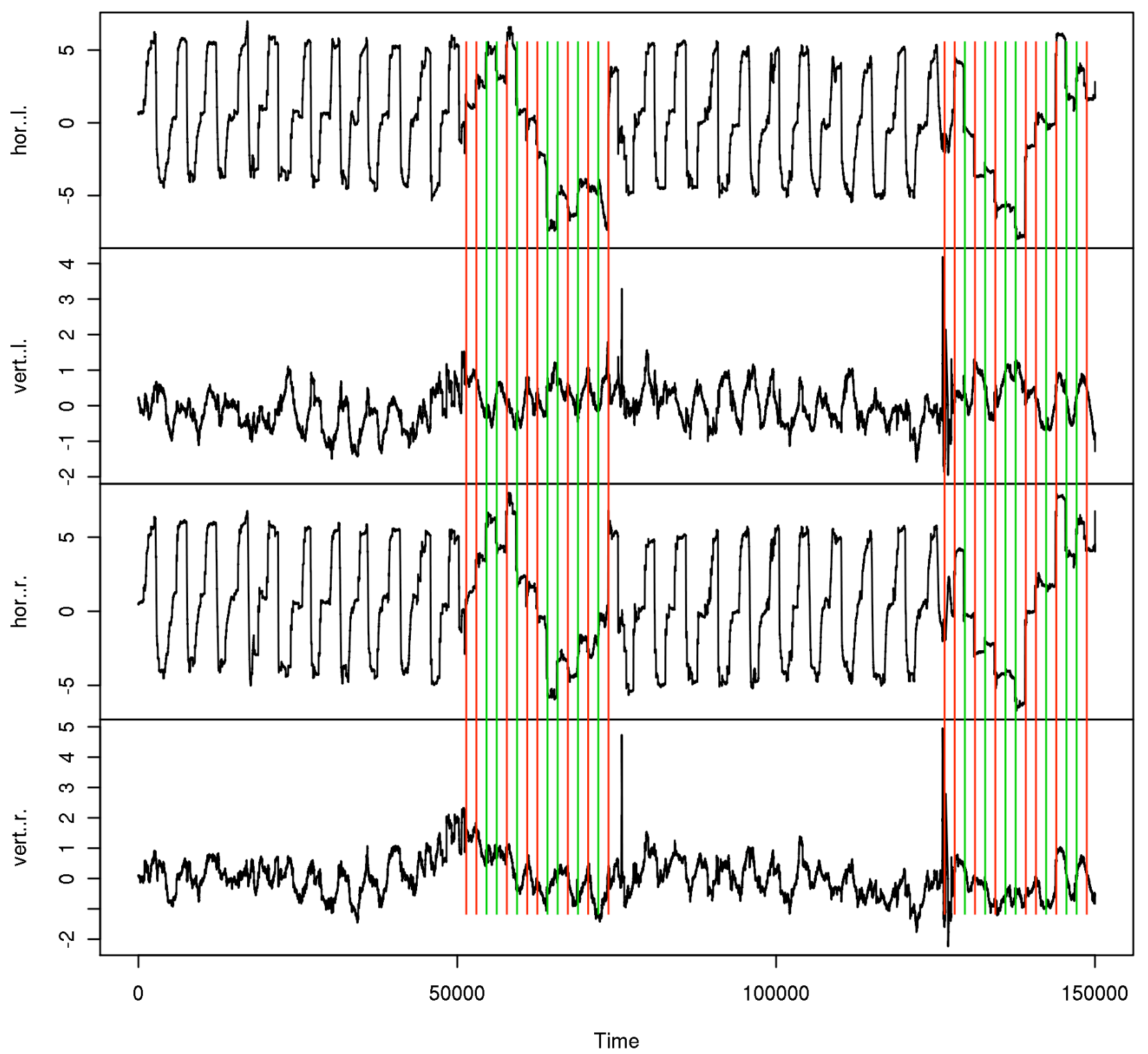

Figure 1. Data sample from one trial. Monocular data phases are marked red for right, green for left eye.

Monocular phases. At two times during the experiment, calibration phases with monocular fixation targets were inserted. At 7 calibration positions in a horizontal line in 3 degree steps, a fixation target was presented in random interleaved order to either the left or right eye. This monocular calibration target was a light square of initial size $10 \times 10$ pixels at pixel size $0.282 \mathrm{~mm}$ shrinking every $100 \mathrm{~ms}$ by one pixel size. After $1000 \mathrm{~ms}$ it changed to a cross $(+)$ of $3 \times 3$ pixel size, and stayed visible for another $400 \mathrm{~ms}$. The screen image for the other eye was blank. The subjects were asked to make a saccade to the target and to fixate the cross as accurately as possible.

Subjects and trial blocks. The 25 subjects were aged 19-57 years (mean 30, SD 14). 6 of them wore refractive corrections, visual acuity was generally 1.0 or better in each eye, only one had 1.25 and 0.63 in the left and right 
eye. All subjects were tested for normal binocular vision. The experiments were undertaken with the understanding and written consent of the subjects, and the procedures were approved by our ethics review board. With respect to microsaccades, the subjects are considered naive, including the participating authors. Figure 1 shows the data time series from one of those trials. For the calibration phases, the stimulated eye is marked in the corresponding subseries. For each subject, 12 blocks of data were taken, each of which contained the 2 calibration phases and the last 10 experimental cycles, i.e. 30 (10 in the single target variant) subjective fixation disparity judgements, before each calibration.

\section{Collected data}

For the process of saccade detection, we follow the main procedure of Engbert and Kliegl (2003b). For each eye and trial, an individual velocity threshold is determined, which depends mainly on measurement noise, microtremor and drifts present in the data. Though their procedure accounted for possible anisotropy in this level by determining it in horizontal and vertical direction separately, we preferred to use just one isotropic value for the velocity magnitude. It is worth mentioning that, generally, the noise level in the vertical measurements is higher than in the horizontal ones; this is partly due to the instrument resolution, which is only half as high in the vertical than in the horizontal direction. Any two-dimensional velocity whose magnitude exceeds a multiple of 6 times of the median for at least 3 consecutive observations, is considered as indicating a candidate for a saccade. For each measurement block, we built up two lists (one for each eye) consisting of the times of saccade start, peak velocity and saccade end, the saccade widths and the pair of measured velocities for both eyes at the time when the velocity of the eye from which the measurement was taken reached its maximum. These two lists are joined together by looking for overlapping start-end intervals, giving a single double-width list of either matched or unmatched saccade data. If a match was possible, the saccade is considered clearly binocular, if the match failed, it is possibly a monocular saccade which will be called unmatched in the further text to distinguish this attribution for single saccades from the attribution of ocularity for the stimulation condition. Note that for any eye the velocity measured in the fellow eye may be higher or lower than the peak velocity, because the time at which the peak velocity occurs may differ, but for matched saccades it is always bounded by the peak velocity of the fellow eye.
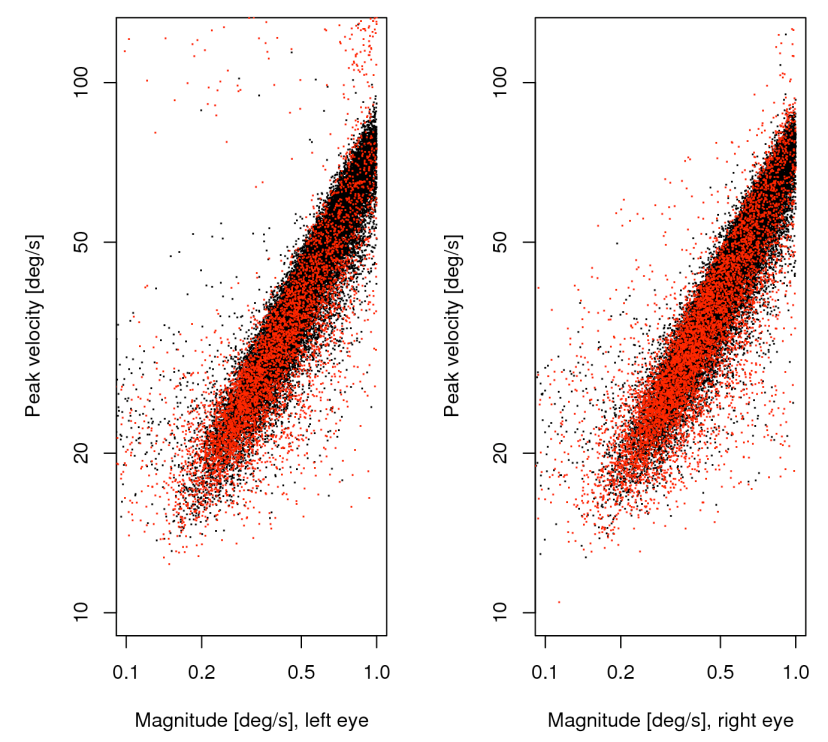

Figure 2. Main sequence of small saccades, unmatched (red) plotted over matched (black). The left and right panels show the data from the respective eye.

\section{Results}

\section{General description of the data set}

We take all saccades up to 1 degree as potential microsaccades. Due to the setup of our experiment only saccades of 3 or more degrees are caused by the experimental task and the calibration process. At a first look, most of the findings on the combined list are consistent with those reported by Engbert and Kliegl (2003b). From 25 participants and 12 measurement blocks we collected 90716 saccade candidates, 38610 of which were less wide than 1 degree, and 11349 of the latter $(34 \%)$ were monocular in the sense that the matching process failed. The main sequence of these data is shown in figure 2, separately for the eyes, with matched saccades in black, unmatched in red. The directions of all microsaccades were distributed with a big horizontal and smaller vertical peak, whereas the vertical peak exceeded the horizontal in the unmatched subset. Substantial numbers of oblique microsaccades were found only in the upper outside quadrants for this subset. Saccade width was distributed with a single peak somewhat below half a degree. This property ensures that the selection made does not split apart a big number of similar cases, though it does not ensure that all selected saccades are microsaccades in the strict sense. It is to be expected that the larger ones are still more likely voluntary non-fixational saccades, and the smaller ones involuntary proper microsaccades. 

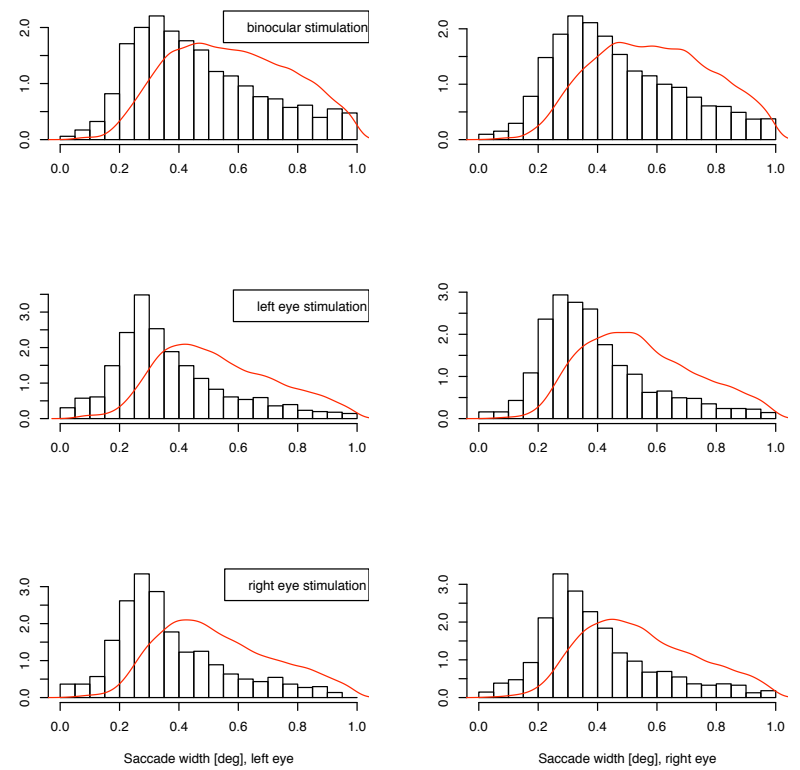

Figure 3. Distribution of small unmatched saccade widths. The red line shows the density for matched saccades. Horizontal: left and right eye, from top to bottom: binocular, monocular to left eye, monocular to right eye presentations.
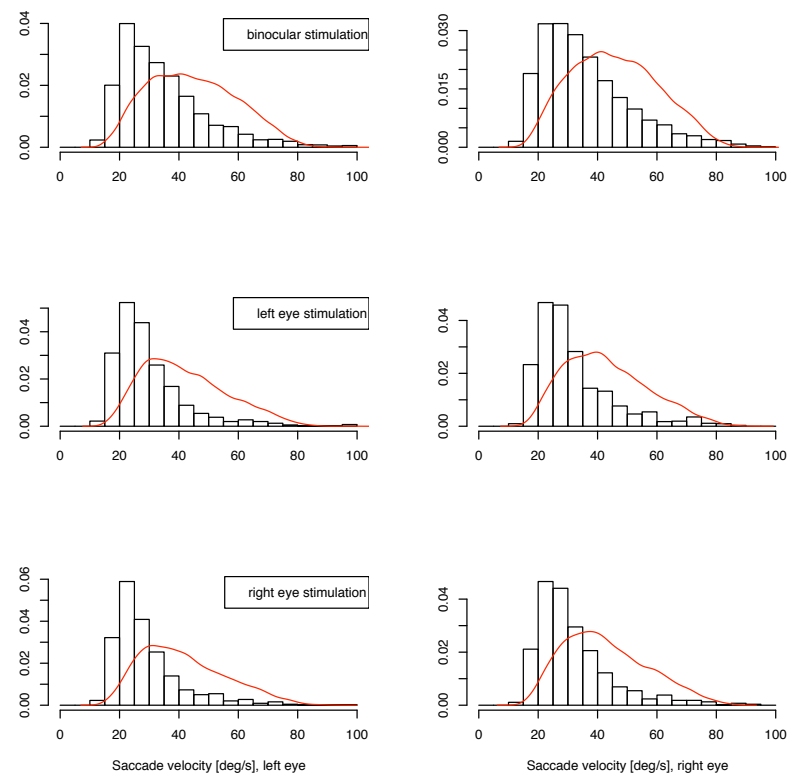

Figure 4. Distribution of small unmatched saccade velocities. The red line shows the density for matched saccades. Horizontal: left and right eye, from top to bottom: binocular, monocular to left eye, monocular to right eye presentations.
Kloke, W. B., Jaschinski, W., \& Jainta, S. (2009) Microsaccades under monocular viewing conditions
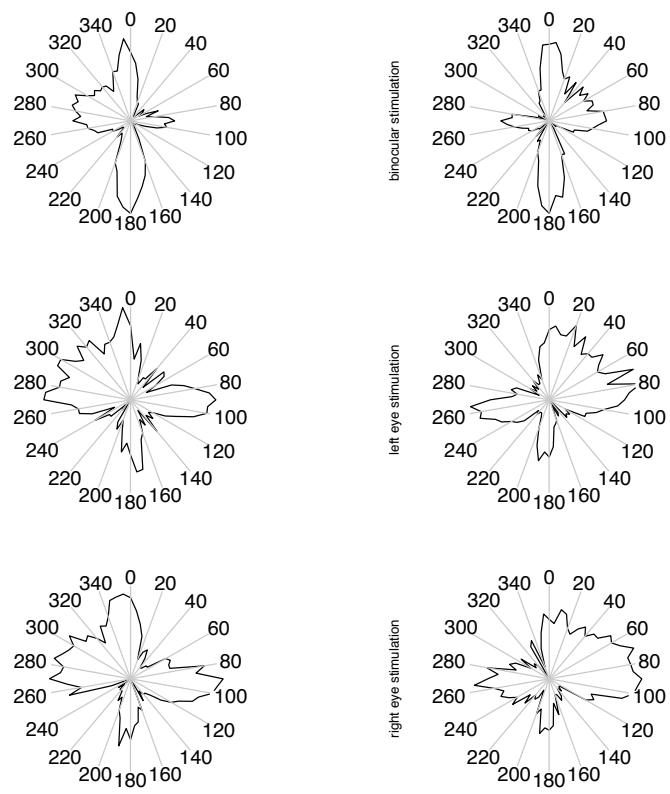

Figure 5. Distribution of small unmatched saccade directions, in natural orientation of viewing direction. On the radial scale the square root of the raw frequencies is plotted, so the area of a sector is indicating the relative frequency of direction.
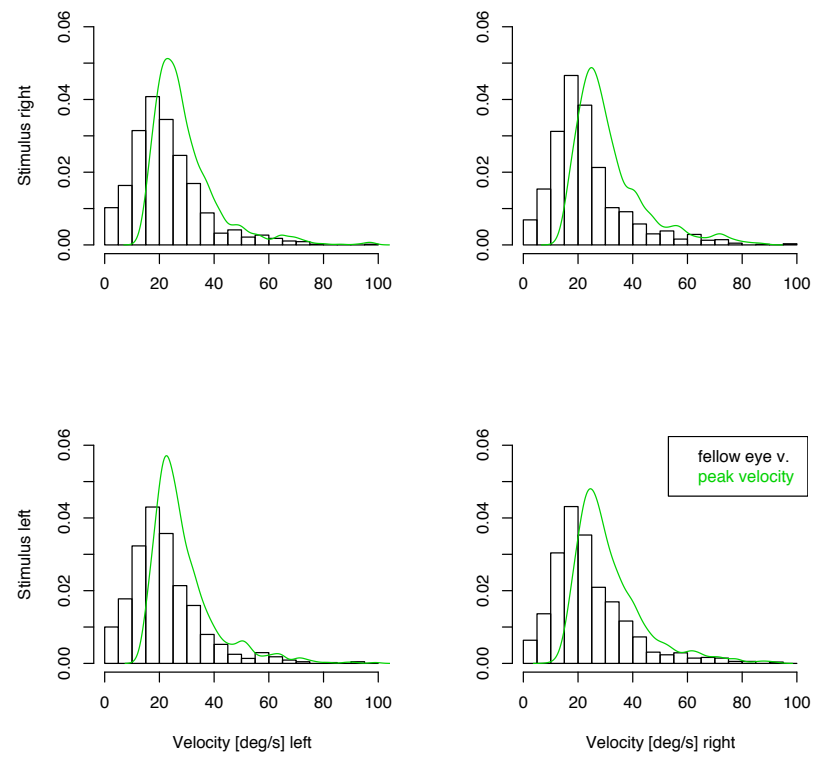

Figure 6. Distribution of velocities of the contralateral eye in small unmatched saccades. The green line shows the density of peak velocities of the eye where the saccade has been detected. Top: Left eye, Bottom: Right eye. 


\section{Distribution of small saccade variables vs. condi- tions}

The data are grouped as those from the two monocular calibration phases in each measurement block, and one of the experimental conditions in the binocular phases. We reported in (Jaschinski et al., 2009), that regarding binocular coordination, the data indeed do show a different pattern between the binocular experiment phase and the monocular calibration phases. In the binocular stimulation context, the eyes converge to the required vergence angle within less than half a degree, whereas during monocular calibration the vergence adopted an inter-individually different, but intra-individually stable, vergence state with a range of several degrees, which is identified as the subject's heterophoria. In the present paper, we are investigating possible interactions for microsaccade data (1) for the monocular vs. binocular stimulation condition and (2) whether the eye whose measurement is considered is the same as the fixating eye or not, at monocular stimulation. Available for this analysis are the saccade's width, velocity and direction for both eyes in the matched cases. For saccades which are not matched in the joining process, the velocity of the fellow eye is available for comparison instead of the unavailable full set of saccade parameters. Due to the conditions of our experiment, we cannot determine a saccade rate, because this is influenced by the presence of intentional saccades. In figures 3 and 4, we present our data as histograms of the measured variables, separately for the conditions "binocular target", "monocular target on left" and "monocular target on right" in this order from top to bottom, and measurement on left and right eye horizontally. For comparison, the corresponding density for the matched case is shown as a continuous line in red. For the direction measurements in figure 5, we use the square root of the raw frequencies to make the area of circular segments proportional to the frequency. For the interpretation of direction data, it should be noted that the measurement error for this variable is inversely related to the velocity magnitude, so the direction of small saccades is measured with less certainty than that of larger ones. A subset of the upper 3 quartiles shows similar distribution as the full set for our data.

If there is a monocular microsaccade generation mechanism and it is modulated by the visual content of the stimulus presentation, we might find some statistical interaction in the observations where the measured eye is the fixating eye or the other one. Such an interaction would be apparent in the lower 4 panels of figure 3, 4 and 5 over cross, for the distribution of saccade widths, peak velocities and saccade directions, but contrarily to the assumption, the distributions are very similar in all cases. Further, in the unmatched subset, no interaction is apparent in the distribution of the velocities in the contralateral eye (figure 6), where
Table 1

Quantiles of saccadic vergence change (in degree) of matched small saccades

\begin{tabular}{llllll} 
& $0 \%$ & $20 \%$ & $50 \%$ & $80 \%$ & $100 \%$ \\
\hline binocular & 0.000 & 0.063 & 0.117 & 0.193 & 1.523 \\
right eye & 0.002 & 0.061 & 0.109 & 0.176 & 1.317 \\
left eye & 0.001 & 0.063 & 0.110 & 0.174 & 0.879
\end{tabular}

no saccade has been detected. In all conditions the distributions of these velocities are similar, too. They are broader distributed than the saccade peak velocities with peaks at lower velocites, and there are some, but not many, low velocities present. This means that in most of the cases where saccades have been detected only in one eye, the velocity of the other eye was quite high, which might indicate an erroneously missing detection.

\section{Distribution of saccadic vergence change}

Possible reasons for such misses of matching microsaccades may be that saccades are not always exactly conjugate, and the threshold levels are not identical for the eyes. The inherent risk of overfitting if the number of threshold parameters used is too high, was the reason why we preferred not to use anisotropic threshold values. The statistics of monocular detected microsaccades may be explained by a coincidence of saccadic vergence change and small saccade velocity near the detection threshold. The pair of velocities could exceed the threshold in one of the eyes and not reach it in the other. To check for this, we computed the vergence change as the difference of saccade width measurements between the eyes for matched saccades. The distribution of vergence change, see table 1 , shows that only $50 \%$ of them shift the vergence less than 0.1 degree, and $20 \%$ shift the vergence more than 0.174 degree. According to the main sequence relation between saccade width and velocity a similar spread between the corresponding velocities has to be taken into account. It is therefore no surprise that smaller saccades are more likely affected. For small vertical saccades the effect is enlarged by the higher noise level present in the vertical measurements. The slightly higher level of vergence change in the binocularly presented cases may be due either to the larger saccades or the higher demand for vergence control, but this cannot be decided from the data.

\section{Discussion}

We compared data describing microsaccades from an experiment with two conditions: (1) In the binocular condition, both eyes fixated the targets. This is the natural test condition in previous studies. (2) In the monocular condition only one of the eyes was 
presented with a fixation target while the fellow eye viewed a blank field without any fixation target. We did not find support for the notion of a difference between these two situations with respect to the statistical properties of microsaccade parameters. Though the microsaccade detection algorithm does not always detect them in both eyes we argue that it still seems more probable that this a weakness of the detection procedure than a real physiological phenomenon. At low saccade widths we observed substantial vergence shifts due to non-perfect conjugateness of saccades which may well account for the missing detection of some microsaccade pairs. In both cases of monocular and binocular stimulation, microsaccades behave just as if the saccade activation is governed by Hering's law and the saccadic vergence shift is randomly varying in a similar range. Consistently with this explanation, the distribution of matched and unmatched small saccades is different but uniformly in both types of experimental conditions. There may be cases left, where a microsaccade is detected in one eye, and the direction change in the fellow eye is really too small to be explained by these arguments alone. For these rare cases, it still may be preferable to consider them as disconjugate binocular saccades, where the saccade width happens to be very small in one of the eyes, than to postulate a specific monocular microsaccade generation mechanism.

The large number of small saccades detected in only one of the eyes using automatic algorithms leads to a serious decision problem, when the microsaccade rate is to be estimated. Either there is a genuine monocular saccade generation mechanism which warrants a distinct handling, or the true microsaccade rate is likely to be underestimated, if monocularly detected saccades are simply discarded. For relative saccade rates between experimental conditions, the effect may be more limited, as we did not find effects on the other statistical properties. As long as the question of the existence of truely monocular saccades is not positively answered, it seems more conservative to include them or to report both results, with and without inclusion. At least, the effect of the decision should be monitored continuously to gain more evidence about this problem.

\section{References}

Bridgeman, B., \& Palca, J. (1980). The role of microsaccades in high acuity observational tasks. Vision Research, 20, 813817.

Engbert, R., \& Kliegl, R. (2001). Mathematical models fo eye movement in reading: A possible role for autonomous accades. Biological Cybernetics, 85, 77-87.

Engbert, R., \& Kliegl, R. (2003a). Binocular coordination in microsaccades. In J. Hyönä, R. Radach, \& H. Deubel (Eds.), The mind's eye: Cognitive and applied aspects of eye movement research (p. 103-117). Oxford: Elsevier.

Engbert, R., \& Kliegl, R. (2003b). Microsaccades uncover the orientation of covert attention. Vision Research, 43, 10351045.
Filin, V. A. (1997). Automation of saccades. Perception, 26 ECVP Abstract Supplement, 96.

Filin, V. A., \& Ananin, V. F. (1973). Involuntary eye movements with and without fixed point. Physiologichskiy jurnal SSSR, 59(4), 547-550.

Hafed, Z. M., Goffart, L., \& Krauzlis, R. J. (2009). A Neural mechanism for microsaccade generation in the primate superior colliculus. Science, 323, 940.

Jaschinski, W., Kloke, W. B., \& Jainta, S. (2009). Vergence errors during short and steady fixations measured with objective and subjective methods. manuscript, in preparation.

Kadyrov, A., \& Petrou, M. (2004). Reverse Engineering the human vision system: a possible explanation for the role of microsaccades. In J. Kittler (Ed.), Proceedings of the 17th International Conference on Pattern Recognition, Aug. 23-26 (Vol. 4, p. 64-67). Los Alamitos: IEEE Computer Soc.

Kowler, E., \& Steinman, R. M. (1980). Small saccades serve no useful purpose: Reply to a letter by R. W. Ditchburn. Vision Research, 20, 273-276.

Krauskopf, J., Cornsweet, T. N., \& Riggs, L. (1960). Analysis of eye movements during monocular and binocular fixation. Journal of the Optical Society of America, 50(6), 572578.

Martinez-Conde, S., \& Macknik, S. L. (2007). Windows on the Mind. Scientific American, 2007(8), 56-63.

Martinez-Conde, S., Macknik, S. L., \& Hubel, D. H. (2004). The role of fixational eye movements in visual perception. Nature Reviews Neuroscience, 5, 229-240.

Martinez-Conde, S., Macknik, S. L., Troncoso, X. G., \& Dyar, T. A. (2006). Microsaccades counteract visual fading during fixation. Neuron, 49, 297-305.

Mays, L. (1998). Has Hering been hooked? Nature Medicine, 4(8), 889-890.

Møller, F., Laursen, M. L., Tygesen, J., \& Sjølie, A. K. (2002). Binocular quantification and characterization of microsaccades. Graefe's Arch Clin Exp Ophthalmol, 240, 765-770.

Otero-Millan, J., Troncoso, X. G., Macknik, S. L., SerranoPedraza, I., \& Martinez-Conde, S. (2008). Saccades and microsaccades during visual fixation, exploration, and search: Foundations for a common saccadic generator. Journal of Vision, 8(14), 1-18.

Richter, E. M., Engbert, R., \& Kliegl, R. (2008). The perpetual gap paradigm: Evidence for an autonomous timer for saccade generation. manuscript, under revision http://www.agnld.unipotsdam.de/ ralf/EngbertLab/Publications.html.

Rolfs, M., Kliegl, R., \& Engbert, R. (2008). Toward a model of microsaccade generation: The case of microsaccadic inhibition. Journal of Vision, 8(11), 5, 1-23.

Troncoso, X. G., Macknik, S. L., \& Martinez-Conde, S. (2008). Microsaccades counteract perceptual filling-in. Journal of Vision, 8(14), 1-9.

Zhou, W., \& King, W. M. (1998). Premotor commands encode monocular eye movements. Nature, 393, 692-695.

Zuber, B. L., \& Stark, L. (1966). Saccadic suppression: Elevation of visual threshold associated with saccadic eye movements. Experimental Neurology, 16, 65-79.

Zuber, B. L., Stark, L., \& Cook, G. (1965). Microsaccades and the velocity-amplitude relationship for saccadic eye movements. Science, 150, 1459-1460. 